\title{
Some approximation results for a generalization of Szász-Mirakjan operators based on quantum calculus
}

\author{
Reşat Aslan \\ Labor and Employment Agency, Paşabağı Mah. 765. Sk. No: 42/A, 63050, Haliliye, Şanlıurfa, Turkey
}

Received: 5 January2021, Accepted: 23 March 2021

Published online: 17 April 2021.

\begin{abstract}
The main purpose of this article is to discuss some approximation properties for a generalization of Szász-Mirakjan operators based on quantum calculus. We obtain a recurrence formula for the moments and compute the central moments for the first, second and fourth order. Moreover, we examine the uniform convergence by Korovkin's type approximation, the order of approximation with regard to the modulus of continuity on a finite closed set and for the functions belong to the Lipschitz class. Further, we investigate the local approximation as well as the weighted uniform approximation results on unbounded interval. Also, we compute the order of convergence in terms of the weighted modulus of continuity and prove the Voronovskaya-type asymptotic theorem for the related operators. Finally, with the help of Maple software, we give a comparison of the convergence of these operators to the certain function with some graphics.
\end{abstract}

Keywords: Szász-Mirakjan operators, $q$-integers, order of convergence, weighted approximation, Voronovskaya-type asymptotic theorem

\section{Introduction}

In [22,26], Szász and Mirakjan defined and introduced the following linear positive operators

$$
S_{n}(f ; x)=e^{-n x} \sum_{k=0}^{\infty} f\left(\frac{k}{n}\right) \frac{(n x)^{k}}{k !}
$$

where $x \geq 0$ and $f \in C[0, \infty)$.

Recently, many modifications of (1) operators have been introduced by several authors. For instance, Gupta and Pant [17] estimated the order of convergence for the modified Szász-Mirakjan operators. Some approximation results for the modified Szász-Mirakjan operators on the weighted spaces were investigated by Ispir and Atakut [18]. Aral et al. [9] obtained several approximation results for a new construction of the Szász-Mirakjan operators. Ousman and Izgi [24] introduced a new modification of Szász-Mirakjan operators on a closed subintervals of $[0, \infty)$ as below:

$$
N_{n}(f ; x)=e^{-n x} \sum_{k=0}^{\infty} f\left(\frac{k}{n} \frac{n+a}{n+b}\right) \frac{(n x)^{k}}{k !} \quad 0 \leq x<\infty,
$$

where $a, b \in \mathbb{N}$ and $0 \leq a \leq b$.

For the operators given by (2), they estimated the degree of convergence, proved the Voronovskaya-type asymptotic theorem and studied the order of approximation of functions on the class of Lipschitz. 
The quantum calculus ( $q$-calculus) which have many applications in disciplines such as engineering and physics besides mathematics, has been an important research topic since the last century. In recent years, the approximation of functions with linear positive operators thanks to the quantum calculus, has become very intensive research area. The first study on this topic was given by Lupas [20]. He obtained several approximation properties for the generalizations of $q-$ Bernstein polynomials. Also, Phillips [25] established some convergence results and Voronovskaya type asymptotic formula for the most popular generalizations of the $q$-Bernstein polynomials. After [20,25] investigations, the implementing of $q$-calculus on the approximation theory becomes very popular and motivated many authors to apply this technic to some various famous operators. For instance, Aral [7] introduced a new generalization of the $q$-Szász-Mirakjan operators. Ahasan and Mursaleen [4] obtained several approximation outcomes of the generalized Szász-Mirakjan operators via $q$-calculus. Further, the Szász-Mirakjan operators on $q$-parametric were introduced by Mahmudov [21]. Agrawal et al. [3] considered the $q$-Szász-Baskakov sort operators. Mursaleen et al. [23] investigated several approximation features of $q$-Baskakov-Schurer-Szász-Stancu type operators. Also, we refer to the readers some other interesting works see: ([11, $13,1,5,8,10,27,12,2])$.

Now, we give some basic notations and definitions which based on $q$-calculus as set out in [19]. Suppose that $0<q<1$. For all integers $n, l$ such that $n \geq l \geq 0$, the $q$-integer $[n]_{q}$ is given by

$$
[n]_{q}:= \begin{cases}\frac{1-q^{n}}{1-q} & q \neq 1 \\ n, & q=1\end{cases}
$$

The $q$-factorial $[n]_{q}$ ! and $q$-binomial $\left[\begin{array}{l}n \\ l\end{array}\right]_{q}$ are given, respectively as follows:

$$
[n]_{q} !:=\left\{\begin{array} { c l } 
{ [ n ] _ { q } [ n - 1 ] _ { q } . . [ 1 ] _ { q } , } & { n = 1 , 2 , . . } \\
{ 1 , } & { n = 0 }
\end{array} \text { and } \left[\begin{array}{l}
n \\
l]_{q}
\end{array}=\frac{[n]_{q} !}{[l]_{q} ![n-l]_{q} !} .\right.\right.
$$

The $q$-analogues of the exponential function $e^{u}$ are defined as below:

$$
e_{q}(u):=\sum_{l=0}^{\infty} \frac{u^{l}}{[l]_{q} !}=\frac{1}{(1-(1-q) u)_{q}^{\infty}}, \quad|u|<\frac{1}{1-q}, \quad|q|<1
$$

and

$$
E_{q}(u):=\sum_{l=0}^{\infty} \frac{u^{l}}{[l]_{q} !} q^{\frac{l(l-1)}{2}}=(1+(1-q) u)_{q}^{\infty}, u \in \mathbb{R}, \quad|q|<1
$$

where $(1-u)_{q}^{\infty}:=\prod_{s=0}^{\infty}\left(1-q^{s} u\right)$. It is easy to check $e_{q}(u) E_{q}(-u)=1$.

Now, inspired by all above mentioned studies, we construct a new generalization of the $q$-Szász-Mirakjan operators as below:

$$
R_{n, q, a, b}(f ; x):=E_{q}\left(-[n]_{q} x\right) \sum_{k=0}^{\infty} \mu_{n, k}(q ; x) f\left(\frac{[k]_{q}}{[n]_{q}} \frac{[n+a]_{q}}{[n+b]_{q} q^{k-2}}\right), n \in \mathbb{N},
$$

where $\mu_{n, k}(q ; x)=q^{\frac{k(k-1)}{2}} \frac{\left([n]_{q} x\right)^{k}}{[k]_{q} !}, x \in[0, \infty), f \in C[0, \infty), a, b \in \mathbb{N}, 0 \leq a \leq b$.

For $0<q<1$ and $x \geq 0$, it is easy to see that $\mu_{n, k}(q ; x) \geq 0$. Furthermore,

$$
E_{q}\left(-[n]_{q} x\right) \sum_{k=0}^{\infty} \mu_{n, k}(q ; x)=E_{q}\left(-[n]_{q} x\right) \sum_{k=0}^{\infty} q^{\frac{k(k-1)}{2}} \frac{\left([n]_{q} x\right)^{k}}{[k]_{q} !}=1 .
$$

It can be seen that the operators given by (3) are linear and positive. If $q=1$ in (3), then (3) becomes to (2). Also if $a=b, q=1$, in (3), then (3) reduces to (1). 
The rest of this work is organized as follows: In section 2, we compute some moments and central moments. Also, we show the uniform convergence of these operators on the interval $[0, A]$, where $A>0$. In section 3 , we evaluate the order of convergence by means of the modulus of continuity, for the functions belong to Lipschitz class and Peetre's K-functional, respectively. In section 4, we compute the order of convergence with the help the weighted modulus of continuity. In section 5, we present the Voronovskaya-type asymptotic theorem. Finally, by using Maple software, we compare the convergence of these operators to the certain function with some graphics.

\section{Main results}

Lemma 1. For the operators defined by (3), the following recurrence formula hold:

$$
R_{n, q, a, b}\left(t^{s+1} ; x\right)=\sum_{u=0}^{s}\left(\begin{array}{l}
s \\
u
\end{array}\right) \frac{x[n+a]_{q}^{s+1-u}}{q^{2 u-s-1}[n]_{q}^{s-u}[n+b]_{q}^{s+1-u}} R_{n, q, a, b}\left(t^{u} ; x\right)
$$

Proof. In view of the equality $[k]_{q}=q^{k-1}+[k-1]_{q}$, we may write

$$
\begin{aligned}
R_{n, q, a, b}\left(t^{s+1} ; x\right) & =E_{q}\left(-[n]_{q} x\right) \sum_{k=0}^{\infty} \frac{[k]_{q}^{s+1}[n+a]_{q}^{s+1}}{q^{(k-2)(s+1)}[n]_{q}^{s+1}[n+b]_{q}^{s+1}} q^{\frac{k(k-1)}{2}} \frac{\left([n]_{q} x\right)^{k}}{[k]_{q} !} \\
& =E_{q}\left(-[n]_{q} x\right) \sum_{k=1}^{\infty} \frac{q[k]_{q}^{s}[n+a]_{q}^{s+1}}{q^{(k-2) s}[n]_{q}^{s}[n+b]_{q}^{s+1} q^{\frac{k(k-1)}{2}}-k+1} \frac{\left([n]_{q} x\right)^{k-1} x}{[k-1]_{q} !} \\
& =E_{q}\left(-[n]_{q} x\right) \sum_{k=1}^{\infty} q \frac{\left(q^{k-1}+[k-1]_{q}\right)^{s}[n+a]_{q}^{s+1}}{q^{(k-2) s}[n]_{q}^{s}[n+b]_{q}^{s+1}} q^{\frac{(k-1)(k-2)}{2}} \frac{\left([n]_{q} x\right)^{k-1} x}{[k-1]_{q} !} \\
& =q E_{q}\left(-[n]_{q} x\right) \sum_{k=1}^{\infty} \sum_{u=0}^{s}\left(\begin{array}{l}
s \\
u
\end{array}\right) q^{(k-1)(s-u)}[k-1]_{q}^{u} \frac{q^{\frac{(k-1)(k-2)}{2}}}{q^{(k-2) s}[n]_{q}^{s}} \frac{[n+a]_{q}^{s+1}}{[n+b]_{q}^{s+1}} \frac{\left.(n]_{q} x\right)^{k-1} x}{[k-1]_{q} !} \\
& =q E_{q}\left(-[n]_{q} x\right) \sum_{u=0}^{s}\left(\begin{array}{l}
s \\
u
\end{array}\right) \frac{[n+a]_{q}^{s-u+1}}{q^{2 u-s}[n]_{q}^{s-u}[n+b]_{q}^{s-u+1}} \sum_{k=1}^{\infty} \frac{[k-1]_{q}^{u}[n+a]_{q}^{u}}{q^{(k-3) u}[n]_{q}^{u}[n+b]_{q}^{u}} q^{\frac{(k-1)(k-2)}{2}} \frac{x\left([n]_{q} x\right)^{k-1}}{[k-1]_{q} !} \\
& =q E_{q}\left(-[n]_{q} x\right) \sum_{u=0}^{s}\left(\begin{array}{l}
s \\
u
\end{array}\right) \frac{x+a]_{q}^{s-u+1}}{q^{2 u-s}[n]_{q}^{s-u}[n+b]_{q}^{s-u+1}} \sum_{k=0}^{\infty} \frac{[k]_{q}^{u}[n+a]_{q}^{u}}{q^{(k-2) u}[n]_{q}^{u}[n+b]_{q}^{u} q^{\frac{k(k-1)}{2}} \frac{\left([n]_{q} x\right)^{k}}{[k]_{q} !}} \\
& =\sum_{u=0}^{s}\left(\begin{array}{l}
s \\
u
\end{array}\right) \frac{x[n+a]_{q}^{s+1-u}}{q^{2 u-s-1}[n]_{q}^{s-u}[n+b]_{q}^{s+1-u} R_{n, q, a, b}\left(t^{u} ; x\right)}
\end{aligned}
$$

which gives the proof of Lemma 1.

Lemma 2. For the operators defined by (3), the following equalities are satisfied:

$$
\begin{gathered}
R_{n, q, a, b}(1 ; x)=1, \\
R_{n, q, a, b}(t ; x)=q x \frac{[n+a]_{q}}{[n+b]_{q}},
\end{gathered}
$$




$$
\begin{gathered}
R_{n, q, a, b}\left(t^{2} ; x\right)=\left(q x^{2}+\frac{q^{2}}{[n]_{q}} x\right) \frac{[n+a]_{q}^{2}}{[n+b]_{q}^{2}} \\
R_{n, q, a, b}\left(t^{3} ; x\right)=\left(x^{3}+\frac{\left(2 q^{2}+q\right)}{[n]_{q}} x^{2}+\frac{q^{3}}{[n]_{q}^{2}} x\right) \frac{[n+a]_{q}^{3}}{[n+b]_{q}^{3}}, \\
R_{n, q, a, b}\left(t^{4} ; x\right)=\left(\frac{x^{4}}{q^{2}}+\frac{3 q^{2}+2 q+1}{q[n]_{q}} x^{3}+\frac{3 q^{3}+3 q^{2}+q}{[n]_{q}^{2}} x^{2}+\frac{q^{4}}{[n]_{q}^{3}} x\right) \frac{[n+a]_{q}^{4}}{[n+b]_{q}^{4}}
\end{gathered}
$$

Proof. Taking (5) into account, then the proof of each equality can be obtained by similar method, thus we will only give the proof of the last two equalities.

$$
\begin{aligned}
R_{n, q, a, b}\left(t^{3} ; x\right) & =\frac{x}{q} \frac{[n+a]_{q}}{[n+b]_{q}} R_{n, q, a, b}\left(t^{2} ; x\right)+\frac{2 q x}{[n]_{q}} \frac{[n+a]_{q}^{2}}{[n+b]_{q}^{2}} R_{n, q, a, b}(t ; x)+\frac{q^{3}}{[n]_{q}^{2}} x \frac{[n+a]_{q}^{3}}{[n+b]_{q}^{3}} R_{n, q, a, b}(1 ; x) \\
& =\frac{x}{q} \frac{[n+a]_{q}}{[n+b]_{q}}\left[\left(q x^{2}+\frac{q^{2}}{[n]_{q}} x\right) \frac{[n+a]_{q}^{2}}{[n+b]_{q}^{2}}\right]+\frac{2 q x}{[n]_{q}} \frac{[n+a]_{q}^{2}}{[n+b]_{q}^{2}}\left[q x \frac{\left.[n+a]_{q}\right]}{[n+b]_{q}}\right]+\frac{q^{3}}{[n]_{q}^{2}} x \frac{[n+a]_{q}^{3}}{[n+b]_{q}^{3}} \\
& =\left(x^{3}+\frac{\left(2 q^{2}+q\right)}{[n]_{q}} x^{2}+\frac{q^{3}}{[n]_{q}^{2}} x\right) \frac{[n+a]_{q}^{3}}{[n+b]_{q}^{3}}
\end{aligned}
$$

and

$$
\begin{aligned}
R_{n, q, a, b}\left(t^{4} ; x\right) & =\frac{x}{q^{2}} \frac{[n+a]_{q}}{[n+b]_{q}} R_{n, q, a, b}\left(t^{3} ; x\right)+\frac{3 x}{[n]_{q}} \frac{[n+a]_{q}^{2}}{[n+b]_{q}^{2}} R_{n, q, a, b}\left(t^{2} ; x\right)+\frac{3 q^{2} x}{[n]_{q}^{2}} \frac{[n+a]_{q}^{3}}{[n+b]_{q}^{3}} R_{n, q, a, b}(t ; x)+\frac{q^{4} x}{[n]_{q}^{3}} \frac{[n+a]_{q}^{4}}{[n+b]_{q}^{4}} R_{n, q, a, b}(1 ; x) \\
& =\frac{x}{q^{2}} \frac{[n+a]_{q}}{[n+b]_{q}}\left[\left(x^{3}+\frac{\left(2 q^{2}+q\right)}{[n]_{q}} x^{2}+\frac{q^{3}}{[n]_{q}^{2}} x\right) \frac{[n+a]_{q}^{3}}{[n+b]_{q}^{3}}\right]+\frac{3 x}{[n]_{q}} \frac{[n+a]_{q}^{2}}{[n+b]_{q}^{2}}\left[\left(q x^{2}+\frac{q^{2}}{[n]_{q}} x\right) \frac{[n+a]_{q}^{2}}{[n+b]_{q}^{2}}\right] \\
& +\frac{3 q^{2} x}{[n]_{q}^{2}} \frac{[n+a]_{q}^{3}}{[n+b]_{q}^{3}}\left[q x \frac{[n+a]_{q}}{[n+b]_{q}}\right]+\frac{q^{4} x}{[n]_{q}^{3}} \frac{[n+a]_{q}^{4}}{[n+b]_{q}^{4}} \\
& =\left(\frac{x^{4}}{q^{2}}+\frac{3 q^{2}+2 q+1}{q[n]_{q}} x^{3}+\frac{3 q^{3}+3 q^{2}+q}{[n]_{q}^{2}} x^{2}+\frac{q^{4}}{[n]_{q}^{3}} x\right) \frac{[n+a]_{q}^{4}}{[n+b]_{q}^{4}} .
\end{aligned}
$$

Corollary 1. As a consequence Lemma 2, we have the following:

$$
\begin{aligned}
R_{n, q, a, b}(t-x ; x) & =x\left\{q \frac{[n+a]_{q}}{[n+b]_{q}}-1\right\}, R_{n, q, a, b}\left((t-x)^{2} ; x\right)=x^{2}\left\{q \frac{[n+a]_{q}^{2}}{[n+b]_{q}^{2}}-2 q \frac{[n+a]_{q}}{[n+b]_{q}}+1\right\}+\frac{q^{2} x}{[n]_{q}} \frac{[n+a]_{q}^{2}}{[n+b]_{q}^{2}}, \\
R_{n, q, a, b}\left((t-x)^{4} ; x\right) & =x^{4}\left\{\frac{1}{q^{2}} \frac{[n+a]_{q}^{4}}{[n+b]_{q}^{4}}-4 \frac{[n+a]_{q}^{3}}{[n+b]_{q}^{3}}+6 q \frac{[n+a]_{q}^{2}}{[n+b]_{q}^{2}}-4 q \frac{[n+a]_{q}}{[n+b]_{q}}+1\right\} \\
& +\frac{x^{3}}{[n]_{q}}\left\{\frac{3 q^{2}+2 q+1}{q} \frac{[n+a]_{q}^{4}}{[n+b]_{q}^{4}}-4\left(2 q^{2}+q\right) \frac{[n+a]_{q}^{3}}{[n+b]_{q}^{3}}+6 q^{2} \frac{[n+a]_{q}^{2}}{[n+b]_{q}^{2}}\right\} \\
& +\frac{x^{2}}{[n]_{q}^{2}}\left\{\left(3 q^{3}+3 q^{2}+q\right) \frac{[n+a]_{q}^{4}}{[n+b]_{q}^{4}}-4 q^{3} \frac{[n+a]_{q}^{3}}{[n+b]_{q}^{3}}\right\}+\frac{q^{4} x}{[n]_{q}^{3}} \frac{[n+a]_{q}^{4}}{[n+b]_{q}^{4}} .
\end{aligned}
$$


Remark. It can be seen that for a fixed $q \in(0,1), \lim _{n \rightarrow \infty}[n]_{q}=\frac{1}{1-q}$ as $n \rightarrow \infty$. In order to provide the results, we get the sequence $q:=\left(q_{n}\right)$ such that $0<q_{n}<1, \lim _{n \rightarrow \infty} q_{n}=1, \lim _{n \rightarrow \infty} \frac{1}{[n]_{q_{n}}}=0$ as $n \rightarrow \infty$.

In the next theorem, in order to get the uniform convergence of the operators given by (3), we take for constant $A>0$, a closed finite interval $[0, A]$ the subinterval of $[0, \infty)$. As it is known, the space $C[0, A]$ denotes the real-valued continuous functions on $[0, A]$ and it is equipped with the norm $\|f\|_{C[0, A]}=\sup _{x \in[0, A]}|f(x)|$.

Theorem 1. Suppose that $q:=\left(q_{n}\right)$ such that $0<q_{n}<1, \lim _{n \rightarrow \infty} q_{n}=1, \lim _{n \rightarrow \infty} \frac{1}{[n]_{q_{n}}}=0$ as $n \rightarrow \infty$ and $A>0$. Then for all $f \in C[0, \infty)$, the operators given by (3) converges to $f$ uniformly on $[0, A]$.

Proof. Taking into account the Bohman-Korovkin theorem given by [14], then we have to show that the operators given by (3) converges to $f$ uniformly on $[0, A]$. By (6), it is clear that

$$
\lim _{n \rightarrow \infty}\left\|R_{n, q_{n}, a, b}(1)-1\right\|_{C[0, A]}=0 .
$$

From (7), one has

$$
\lim _{n \rightarrow \infty}\left\|R_{n, q_{n}, a, b}(t)-x\right\|_{C[0, A]}=\lim _{n \rightarrow \infty} \max _{x \in[0, A]}\left|q_{n} x \frac{[n+a]_{q_{n}}}{[n+b]_{q_{n}}}-x\right| \leq \lim _{n \rightarrow \infty} A\left|q_{n} \frac{[n+a]_{q_{n}}}{[n+b]_{q_{n}}}-1\right|=0 .
$$

Proceeding similarly, by (8)

$$
\begin{aligned}
\lim _{n \rightarrow \infty}\left\|R_{n, q_{n}, a, b}\left(t^{2}\right)-x^{2}\right\|_{C[0, A]} & =\lim _{n \rightarrow \infty} \max _{x \in[0, A]}\left|\left(q_{n} x^{2}+\frac{q_{n}^{2} x}{[n]_{q_{n}}}\right) \frac{[n+a]_{q_{n}}^{2}}{[n+b]_{q_{n}}^{2}}-x^{2}\right| \\
& \leq \lim _{n \rightarrow \infty} A^{2}\left|q_{n} \frac{[n+a]_{q_{n}}^{2}}{[n+b]_{q_{n}}^{2}}-1\right|+\lim _{n \rightarrow \infty} A\left|\frac{q_{n}^{2}}{[n]_{q_{n}}} \frac{[n+a]_{q_{n}}^{2}}{[n+b]_{q_{n}}^{2}}\right|=0 .
\end{aligned}
$$

Hence, this completes the proof.

\section{Order of convergence and local approximation}

In this section, we evaluate the order of convergence with regard to the modulus of continuity and obtain the local approximation results for the operators given by (3). Let $C_{B}[0, \infty)$ represent the space fof all real-valued continuous and bounded functions $h$ on $[0, \infty)$. On $C_{B}[0, \infty)$, the norm is given as $\|h\|=\sup _{x \in[0, \infty)}|h(x)|$. Moreover, the Peetre's $\mathrm{K}$-functional is defined by

$$
K_{2}(h, \eta)=\inf _{g \in C_{B}^{2}}\left\{\|h-g\|+\eta\left\|g^{\prime \prime}\right\|\right\},
$$

where $\eta>0$ and $C_{B}^{2}=\left\{g \in C_{B}[0, \infty): g^{\prime}, g^{\prime \prime} \in C_{B}[0, \infty)\right\}$. In view of [15], there consist an absolute constant $C>0$ such that

$$
K_{2}(h ; \eta) \leq C \omega_{2}(h ; \sqrt{\eta}), \quad \eta>0,
$$

where

$$
\omega_{2}(h ; \eta)=\sup _{0<a \leq \eta} \sup _{x \in[0, \infty)}|h(x+2 a)-2 h(x+a)+h(x)|
$$

is the second-order modulus of smoothness of the function $h \in C_{B}[0, \infty)$. Further, by $\omega(h ; \eta):=\sup _{0<a \leq \eta}$ sup $|h(x+a)-h(x)|$ we state the ordinary modulus of continuity of $h \in C_{B}[0, \infty)$. Since $\eta>0, \omega(h, \eta)$ has some $x \in[0, \infty)$ useful properties see: [6]. 
Theorem 2. Let $q:=\left(q_{n}\right)$ such that $0<q_{n}<1, \lim _{n \rightarrow \infty} q_{n}=1, \lim _{n \rightarrow \infty} \frac{1}{[n]_{q_{n}}}=0$ as $n \rightarrow \infty$. Then, for all $f \in C[0, \infty)$ and for constant $A>0$, we obtain

$$
\left|R_{n, q_{n}, a, b}(f ; x)-f(x)\right| \leq 2 \omega\left(f ; \sqrt{\eta_{n, q_{n}}(x)}\right),
$$

where $x \in[0, A]$ and $\eta_{n, q_{n}}(x)=R_{n, q_{n}, a, b}\left((t-x)^{2} ; x\right)$.

Proof. Suppose that $f \in C[0, \infty)$ and $x \in[0, A]$. Using the linearity of the operators (3), then

$$
\left|R_{n, q_{n}, a, b}(f ; x)-f(x)\right| \leq\left|\sum_{k=0}^{\infty} \mu_{n, k}\left(q_{n} ; x\right) f\left(\frac{[k]_{q_{n}}}{[n]_{q_{n}}} \frac{[n+a]_{q_{n}}}{[n+b]_{q_{n}} q_{n}^{k-2}}\right)-f(x)\right| \leq \sum_{k=0}^{\infty}\left|f\left(\frac{[k]_{q_{n}}}{[n]_{q_{n}}} \frac{[n+a]_{q_{n}}}{[n+b]_{q_{n}} q_{n}^{k-2}}\right)-f(x)\right| \mu_{n, k}\left(q_{n} ; x\right) .
$$

In view of the following well-known property of modulus of continuity

$$
|f(t)-f(x)| \leq \omega(f ; \eta)\left(\frac{|t-x|}{\eta}+1\right), \eta>0
$$

Then,

$$
\begin{aligned}
\left|R_{n, q_{n}, a, b}(f ; x)-f(x)\right| & \leq \sum_{k=0}^{\infty} \mu_{n, k}\left(q_{n} ; x\right)\left(\frac{\left|\frac{[k]_{q_{n}}}{[n]_{q_{n}}} \frac{[n+a]_{q_{n}}}{[n+b]_{q_{n}} q_{n}^{k-2}}-x\right|}{\eta}+1\right) \omega(f ; \eta) \\
& =\omega(f ; \eta)+\frac{\omega(f ; \eta)}{\eta} \sum_{k=0}^{\infty} \mu_{n, k}\left(q_{n} ; x\right)\left|\frac{[k]_{q_{n}}}{[n]_{q_{n}}} \frac{[n+a]_{q_{n}}}{[n+b]_{q_{n}} q_{n}^{k-2}}-x\right|
\end{aligned}
$$

Utilizing the Cauchy-Schwarz inequality, then

$$
\begin{aligned}
\sum_{k=0}^{\infty} \mu_{n, k}\left(q_{n} ; x\right)\left|\frac{[k]_{q_{n}}}{[n]_{q_{n}}} \frac{[n+a]_{q_{n}}}{[n+b]_{q_{n}} q_{n}^{k-2}}-x\right| & \leq \sum_{k=0}^{\infty}\left\{\mu_{n, k}\left(q_{n} ; x\right)\right\}^{\frac{1}{2}}\left\{\mu_{n, k}\left(q_{n} ; x\right)\left(\frac{[k]_{q_{n}}}{[n]_{q_{n}}} \frac{[n+a]_{q_{n}}}{[n+b]_{q_{n}} q_{n}^{k-2}}-x\right)^{2}\right\}^{\frac{1}{2}} \\
& \leq\left\{R_{n, q_{n}, a, b}\left((t-x)^{2} ; x\right)\right\}^{\frac{1}{2}}
\end{aligned}
$$

Accordingly,

$$
\left|R_{n, q_{n}, a, b}(f ; x)-f(x)\right| \leq\left(1+\frac{1}{\eta} \sqrt{R_{n, q_{n}, a, b}\left((t-x)^{2} ; x\right)}\right) \omega(f ; \eta) .
$$

Consequently, in Corollary 1 on replacing $q$ by a sequence $\left(q_{n}\right)$, it gives $\lim _{n \rightarrow \infty} R_{n, q_{n}, a, b}\left((t-x)^{2} ; x\right)=0$. Choosing $\eta_{n, q_{n}}(x)=$ $R_{n, q_{n}, a, b}\left((t-x)^{2} ; x\right)$ and $\eta=\sqrt{\eta_{n, q_{n}}(x)}$, which completes the proof.

Furthermore, we will examine the order of convergence of the operators (3) for a function on Lipschitz class $\operatorname{Lip}_{M}(\zeta)$, where $M>0$ and $0<\zeta \leq 1$. If the inequality $|h(t)-h(x)| \leq M|t-x|^{\zeta},(t, x \in \mathbb{R})$, holds, then one can say a function $h$ is belong to $\operatorname{Lip}_{M}(\zeta)$.

Theorem 3. Let $q:=\left(q_{n}\right)$ such that $0<q_{n}<1, \lim _{n \rightarrow \infty} q_{n}=1, \lim _{n \rightarrow \infty} \frac{1}{[n]_{q_{n}}}=0$ as $n \rightarrow \infty$. For $f \in \operatorname{Lip} p_{M}(\zeta), x \in[0, A]$, A>0 constant, we have

$$
\left|R_{n, q_{n}, a, b}(f ; x)-f(x)\right| \leq M\left(\eta_{n, q_{n}}(x)\right)^{\frac{\zeta}{2}}
$$

where $\eta_{n, q_{n}}(x)$ is given by Theorem 2. 
Proof. Suppose that $f \in \operatorname{Lip}_{M}(\zeta)$. From the definition of (3) operators, then we obtain

$$
\begin{aligned}
\left|R_{n, q_{n}, a, b}(f ; x)-f(x)\right| & \leq \sum_{k=0}^{\infty} \mu_{n, k}\left(q_{n} ; x\right)\left|f\left(\frac{[k]_{q_{n}}}{[n]_{q_{n}}} \frac{[n+a]_{q_{n}}}{[n+b]_{q_{n}} q_{n}^{k-2}}\right)-f(x)\right| \mu_{n, k}\left(q_{n} ; x\right) \\
& \leq M \sum_{k=0}^{\infty} \mu_{n, k}\left(q_{n} ; x\right)\left|\frac{[k]_{q_{n}}}{[n]_{q_{n}}} \frac{[n+a]_{q_{n}}}{[n+b]_{q_{n}} q_{n}^{k-2}}-x\right|^{\zeta} .
\end{aligned}
$$

Using the Hölder's inequality and choosing $p_{1}=\frac{2}{\zeta}$ and $p_{2}=\frac{2}{2-\zeta}$, one has $\frac{1}{p_{1}}+\frac{1}{p_{2}}=1$. Hence, we get

$$
\begin{aligned}
\left|R_{n, q_{n}, a, b}(f ; x)-f(x)\right| & \leq M \sum_{k=0}^{\infty}\left\{\mu_{n, k}\left(q_{n} ; x\right)\right\}^{\frac{2-\zeta}{2}}\left\{\mu_{n, k}\left(q_{n} ; x\right)\left(\frac{[k]_{q_{n}}}{[n]_{q_{n}}} \frac{[n+a]_{q_{n}}}{[n+b]_{q_{n}} q_{n}^{k-2}}-x\right)^{2}\right\}^{\frac{\zeta}{2}} \\
& \leq M\left\{\sum_{k=0}^{\infty} \mu_{n, k}\left(q_{n} ; x\right)\left(\frac{[k]_{q_{n}}}{[n]_{q_{n}}} \frac{[n+a]_{q_{n}}}{[n+b]_{q_{n}} q_{n}^{k-2}}-x\right)^{\frac{\zeta}{2}}\right\}^{2} \\
& \leq M\left\{R_{n, q_{n}, a, b}\left((t-x)^{2} ; x\right\}^{\frac{\zeta}{2}}=M\left(\eta_{n, q_{n}}(x)\right)^{\frac{\zeta}{2}} .\right.
\end{aligned}
$$

Thus, the proof is completed.

Theorem 4. For all $f \in C_{B}[0, \infty)$ and for $x \in[0, \infty)$, the following inequality holds

$$
\left|R_{n, q, a, b}(f ; x)-f(x)\right| \leq C \omega_{2}\left(f ; \frac{1}{2} \sqrt{\eta_{n, q}(x)}\right)+\omega\left(f ;\left|\beta_{n, q}(x)\right|\right)
$$

where $C>0$ is a constant, $\eta_{n, q}(x)=R_{n, q, a, b}\left((t-x)^{2} ; x\right)$ and $\beta_{n, q}(x)=x\left(q \frac{[n+a]_{q}}{[n+b]_{q}}-1\right)$.

Proof. Let us define the following auxiliary operators:

$$
\stackrel{*}{R}_{n, q, a, b}(f ; x)=R_{n, q, a, b}(f ; x)-f\left(q x \frac{[n+a]_{q}}{[n+b]_{q}}\right)+f(x)
$$

By Corollary 1, it follows $\stackrel{*}{R}, q, a, b(t-x ; x)=0$. From Taylor's expansion formula, then we get

$$
g(t)=g(x)+(t-x) g^{\prime}(x)+\int_{x}^{t}(t-u) g^{\prime \prime}(u) d u, \quad g \in C_{B}^{2}[0, \infty)
$$

After applying $\stackrel{*}{R}_{n, q, a, b}(. ; x)$ to (11), we have

$$
\begin{aligned}
& \stackrel{*}{R}_{n, q, a, b}(g ; x)-g(x)=\stackrel{*}{R}_{n, q, a, b}\left((t-x) g^{\prime}(x) ; x\right)+\stackrel{*}{R}_{n, q, a, b}\left(\int_{x}^{t}(t-u) g^{\prime \prime}(u) d u ; x\right) \\
& =g^{\prime}(x) R_{n, q, a, b}^{*}(t-x ; x)+R_{n, q, a, b}\left(\int_{x}^{t}(t-u) g^{\prime \prime}(u) d u ; x\right)-\int_{x}^{q x}\left(q x \frac{[n+a]_{q}}{\left[n+b_{q}\right.}(n+b]_{q}-u\right) g^{\prime \prime}(u) d u
\end{aligned}
$$




$$
=R_{n, q, a, b}\left(\int_{x}^{t}(t-u) g^{\prime \prime}(u) d u ; x\right)-\int_{x}^{q x \frac{[n+a]_{q}}{[n+b]_{q}}}\left(q x \frac{[n+a]_{q}}{[n+b]_{q}}-u\right) g^{\prime \prime}(u) d u .
$$

It follows from Lemma 2 and by (10), one has

$$
\begin{aligned}
\left|R_{n, q, a, b}(g ; x)-g(x)\right| & \leq R_{n, q, a, b}\left(\left|\int_{x}^{t}(t-u) g^{\prime \prime}(u) d u\right| ; x\right)+\mid \int_{x}^{q x}\left(q x \frac{[n+a]_{q}}{[n+b]_{q}}\left[\begin{array}{l}
{[n+b]_{q}} \\
{[n]^{\prime}}
\end{array}-u\right) g^{\prime \prime}(u) d u \mid\right. \\
& \leq R_{n, q, a, b}\left((t-x)^{2} ; x\right)\left\|g^{\prime \prime}(u)\right\|+\left(q x \frac{[n+a]_{q}}{[n+b]_{q}}-x\right)^{2}\left\|g^{\prime \prime}(u)\right\| .
\end{aligned}
$$

Also, from (8), (10) and considering Corollary 1, then we get

$$
\begin{aligned}
\left|\stackrel{*}{R}_{n, q, a, b}(f ; x)\right| & \leq\left|R_{n, q, a, b}(f ; x)\right|+2\|f\| \\
& \leq\|f\| R_{n, q, a, b}(1 ; x)+2\|f\| \leq 3\|f\| .
\end{aligned}
$$

Using (10) and (12) implies

$$
\begin{aligned}
\left|R_{n, q, a, b}(f ; x)-f(x)\right| & \leq\left|R_{n, q, a, b}(f-g ; x)-(f-g)(x)\right|+|\stackrel{*}{R}, q, a, b(g ; x)-g(x)|+\left|f(x)-f\left(q x \frac{[n+a]_{q}}{[n+b]_{q}}\right)\right| \\
& \leq 4\|f-g\|+\left[R_{n, q, a, b}\left((t-x)^{2} ; x\right)+\left(q x \frac{[n+a]_{q}}{[n+b]_{q}}-x\right)^{2}\right]\left\|g^{\prime \prime}\right\|+\omega\left(f ;\left|q x \frac{[n+a]_{q}}{[n+b]_{q}}-x\right|\right) .
\end{aligned}
$$

Therefore, if we take the infimum on the right hand side over all $g \in C_{B}^{2}[0, \infty)$, from (9) and for constant $C>0, \beta_{n, q}(x)=$ $x\left(q \frac{[n+a]_{q}}{[n+b]_{q}}-1\right)$ and for $\eta_{n, q}(x)=R_{n, q, a, b}\left((t-x)^{2} ; x\right)$, then one has

$$
\left|R_{n, q, a, b}(f ; x)-f(x)\right| \leq 4 K_{2}\left(f ; \eta_{n, q}^{2}(x)\right)+\omega\left(f ;\left|\beta_{n, q}(x)\right|\right) \leq C \omega_{2}\left(f ; \eta_{n, q}(x)\right)+\omega\left(f ;\left|\beta_{n, q}(x)\right|\right)
$$

which gives the proof.

\section{Approximation on weighted spaces}

In this section, we prove Korovkin type approximation theorem on weighted spaces. Also, we estimate the order of convergence of the operators (3) by the help of weighted modulus of continuity.

Let $B_{x^{2}}[0, \infty)$ be the set of all functions $h$ verifying the condition $|h(x)| \leq M_{h}\left(1+x^{2}\right), x \in[0, \infty)$ with constant $M_{h}$, depend only on $h$. We denote by $C_{x^{2}}[0, \infty)$ the set of all continuous functions belonging to $B_{x^{2}}[0, \infty)$ endowed with $\|h\|_{x^{2}}=\sup _{x \in[0, \infty)} \frac{|h(x)|}{1+x^{2}}$ and $C_{x^{2}}^{*}[0, \infty):=\left\{h: h \in C_{x^{2}}[0, \infty), \lim _{x \rightarrow \infty} \frac{|h(x)|}{1+x^{2}}<\infty\right\}$.

Theorem 5. Let $q:=\left(q_{n}\right)$ such that $0<q_{n}<1, \lim _{n \rightarrow \infty} q_{n}=1, \lim _{n \rightarrow \infty} \frac{1}{[n]}=0$ as $n \rightarrow \infty$. Thus, for all $f \in C_{x^{2}}^{*}[0, \infty)$ we obtain

$$
\lim _{n \rightarrow \infty} \sup _{x \in[0, \infty)} \frac{\left|R_{n, q_{n}, a, b}(f ; x)-f(x)\right|}{1+x^{2}}=0 .
$$


Proof. Taking into account the Korovkin type theorem given by Gadzhiev [16]; hence, we have to show that (3) operators satisfy the following condition:

$$
\lim _{n \rightarrow \infty} \sup _{x \in[0, \infty)} \frac{\left|R_{n, q_{n}, a, b}\left(t^{s} ; x\right)-x^{s}\right|}{1+x^{2}}=0, \quad s=0,1,2
$$

Using (6), the first condition in (13) is clear for $s=0$. For $s=1$,by (7), we infer

$$
\sup _{x \in[0, \infty)} \frac{\left|R_{n, q_{n}, a, b}(t ; x)-x\right|}{1+x^{2}}=\left(q_{n} \frac{[n+a]_{q_{n}}}{[n+b]_{q_{n}}}-1\right) \sup _{x \in[0, \infty)} \frac{x}{1+x^{2}}
$$

It follows

$$
\lim _{n \rightarrow \infty} \sup _{x \in[0, \infty)} \frac{\left|R_{n, q_{n}, a, b}(t ; x)-x\right|}{1+x^{2}}=0
$$

Likewise, for $s=2$, from (8), we obtain

$$
\sup _{x \in[0, \infty)} \frac{\left|R_{n, q_{n}, a, b}\left(t^{2} ; x\right)-x^{2}\right|}{1+x^{2}}=\left(q_{n} \frac{[n+a]_{q_{n}}^{2}}{[n+b]_{q_{n}}^{2}}-2 q_{n} \frac{[n+a]_{q_{n}}}{[n+b]_{q_{n}}}+1\right) \sup _{x \in[0, \infty)} \frac{x^{2}}{1+x^{2}}+\left(q_{n}^{2} \frac{[n+a]_{q_{n}}^{2}}{[n]_{q_{n}}[n+b]_{q_{n}}^{2}}\right) \sup _{x \in[0, \infty)} \frac{x}{1+x^{2}}
$$

Hence, we get

$$
\lim _{n \rightarrow \infty} \sup _{x \in[0, \infty)} \frac{\left|R_{n, q_{n}, a, b}\left(t^{2} ; x\right)-x^{2}\right|}{1+x^{2}}=0 .
$$

This completes the proof.

It is known that, if a function $h$ is not uniformly continuous on $[0, \infty)$, the ordinary modulus of continuity $\omega(h ; \eta)$ does not tend to zero, as $\eta \rightarrow 0$. Then, for all $h \in C_{x^{2}}^{*}[0, \infty)$, we take the weighted modulus of continuity $\Omega(h ; \eta)$ as below:

$$
\Omega(h ; \eta)=\sup _{0<a \leq \eta, x \geq 0} \frac{|h(x+a)-h(x)|}{1+(x+a)^{2}} .
$$

Lemma 3. [21]. Let $h \in C_{x^{2}}^{*}[0, \infty)$. Then,

(i) $\Omega(h ; \eta)$ is a monotone increasing function of $\eta$,

(ii) $\lim _{\eta \rightarrow 0^{+}} \Omega(h ; \eta)=0$,

(iii) for any $\alpha \in[0, \infty), \Omega(h ; \alpha \eta) \leq(1+\alpha) \Omega(h ; \eta)$.

Theorem 6. Let $q:=\left(q_{n}\right)$ be the sequence such that $0<q_{n}<1, \lim _{n \rightarrow \infty} q_{n}=1, \lim _{n \rightarrow \infty} \frac{1}{[n]_{q_{n}}}=0$ as $n \rightarrow \infty$. Then, for $f \in C_{x^{2}}^{*}[0, \infty)$, $x \in[0, \infty)$ and sufficiently for large $n$ we obtain

$$
\left|R_{n, q_{n}, a, b}(f ; x)-f(x)\right| \leq C_{3}\left(1+x^{2+\gamma}\right) \Omega\left(f ; \eta_{n}\right),
$$

where $C_{3}>0$ is a constant independent of $f$ and $n$,

$$
\gamma \geq 1, \eta_{n}=\max \left\{\theta_{n}, \vartheta_{n}\right\}, \theta_{n}=q_{n} \frac{[n+a]_{q_{n}}^{2}}{[n+b]_{q_{n}}^{2}}-2 q_{n} \frac{[n+a]_{q_{n}}}{[n+b]_{q_{n}}}+1, \vartheta_{n}=q_{n}^{2} \frac{[n+a]_{q_{n}}^{2}}{[n]_{q_{n}}[n+b]_{q_{n}}^{2}} .
$$

Proof. For $x \in[0, \infty)$ and $t, \eta>0$, using the definition of $\Omega(h ; \eta)$ and by Lemma 3 , we get

$$
|f(t)-f(x)| \leq\left(1+(x+|t-x|)^{2}\right)\left(1+\frac{|t-x|}{\eta_{n}}\right) \Omega(f ; \eta) \leq\left(1+(t+2 x)^{2}\right)\left(1+\frac{|t-x|}{\eta_{n}}\right) \Omega\left(f ; \eta_{n}\right) .
$$


Then, we have

$$
\begin{aligned}
& \left.\left|R_{n, q_{n}, a, b}(f ; x)-f(x)\right| \leq R_{n, q_{n}, a, b}(|f(t)-f(x)| ; x)\right) \\
& \leq\left[R_{n, q_{n}, a, b}\left(\left(1+(t+2 x)^{2}\right) ; x\right)+R_{n, q_{n}, a, b}\left(\left(1+(t+2 x)^{2}\right) \frac{|t-x|}{\eta_{n}} ; x\right)\right] \Omega\left(f ; \eta_{n}\right) .
\end{aligned}
$$

If we apply the Cauchy-Schwarz inequality to the last part of above expression, it gives

$$
R_{n, q_{n}, a, b}\left(\left(1+(t+2 x)^{2}\right) \frac{|t-x|}{\eta_{n}} ; x\right) \leq \sqrt{R_{n, q_{n}, a, b}\left(\left(1+(t+2 x)^{2}\right)^{2} ; x\right)} \sqrt{R_{n, q_{n}, a, b}\left(\frac{|t-x|^{2}}{\eta_{n}^{2}} ; x\right)} .
$$

Hence,

$$
\left|R_{n, q_{n}, a, b}(f ; x)-f(x)\right| \leq \Omega\left(f ; \eta_{n}\right)\left[R_{n, q_{n}, a, b}\left(\left(1+(t+2 x)^{2}\right) ; x\right)+\sqrt{R_{n, q_{n}, a, b}\left(\left(1+(t+2 x)^{2}\right)^{2} ; x\right)} \sqrt{R_{n, q_{n}, a, b}\left(\frac{|t-x|^{2}}{\eta_{n}^{2}} ; x\right)}\right]
$$

In view of Lemma $2, \exists K_{1}>0$ such that $\frac{1}{1+x^{2}} R_{n, q_{n}, a, b}\left(t^{2}+1 ; x\right) \leq K_{1}+1$, then there exists a positive constant $C_{1}$ such that

$$
R_{n, q_{n}, a, b}\left(1+(t+2 x)^{2} ; x\right) \leq C_{1}\left(1+x^{2}\right) .
$$

Analogously,

$$
\frac{1}{1+x^{4}} R_{n, q_{n}, a, b}\left(t^{4}+1 ; x\right) \leq K_{2}+1
$$

we get $\sqrt{R_{n, q_{n}, a, b}\left(\left(1+(t+2 x)^{2}\right)^{2} ; x\right)} \leq C_{2}\left(1+x^{2}\right)$, for some $C_{2}, K_{2}>0$. On the other hand, we have

$$
\sqrt{R_{n, q_{n}, a, b}\left(|t-x|^{2} ; x\right)} \leq \sqrt{\left(q_{n} \frac{[n+a]_{q_{n}}^{2}}{[n+b]_{q_{n}}^{2}}-2 q_{n} \frac{[n+a]_{q_{n}}}{[n+b]_{q_{n}}}+1\right) x^{2}+\left(q_{n}^{2} \frac{[n+a]_{q_{n}}^{2}}{[n]_{q_{n}}[n+b]_{q_{n}}^{2}}\right) x}=\sqrt{\theta_{n} x^{2}+\vartheta_{n} x}
$$

Using (14), one has

$$
\left|R_{n, q_{n}, a, b}(f ; x)-f(x)\right| \leq\left(1+x^{2}\right)\left(C_{1}+\frac{C_{2}}{\eta_{n}} \sqrt{\theta_{n} x^{2}+\vartheta_{n} x}\right) \Omega\left(f ; \eta_{n}\right) .
$$

If we choose $\eta_{n}=\max \left\{\theta_{n}, \vartheta_{n}\right\}$, we have

$\left|R_{n, q_{n}, a, b}(f ; x)-f(x)\right| \leq\left(1+x^{2}\right)\left(C_{1}+C_{2} \sqrt{x^{2}+x}\right) \Omega\left(f ; \eta_{n}\right) \leq C_{3}\left(1+x^{2+\gamma}\right) \Omega\left(f ; \eta_{n}\right)$, for $x \in[0, \infty)$ and sufficiently large $n$

Then, the desired sequel is obtained.

\section{Voronovskaya type asymptotic theorem}

In this section, we will derive the Voronovskaya type asymptotic theorem. First, let's give the lemma below, which we will use in the proof of this theorem.

Lemma 4. Let $q:=\left(q_{n}\right)$ be the sequences such that $0<q_{n}<1, q_{n} \rightarrow 1$ and $q_{n}^{n} \rightarrow c \in[0,1)$, as $n \rightarrow \infty$. Then, for each $x \in[0, \infty)$ the following relations holds:

$$
\begin{gathered}
\lim _{n \rightarrow \infty}[n]_{q_{n}} R_{n, q_{n}, a, b}(t-x ; x)=(c-1) x \\
\lim _{n \rightarrow \infty}[n]_{q_{n}} R_{n, q_{n}, a, b}\left((t-x)^{2} ; x\right)=(1-c) x^{2}+x
\end{gathered}
$$




$$
\lim _{n \rightarrow \infty}[n]_{q_{n}}^{2} R_{n, q_{n}, a, b}\left((t-x)^{4} ; x\right)=3(1-c)^{2} x^{4}+6(1-c) x^{3}+3 x^{2}
$$

Proof. Taking into account Corollary 1, the proof can be obtained easily, so we omitted the details.

Theorem 7. Let $q:=\left(q_{n}\right)$ be the sequences such that $0<q_{n}<1, q_{n} \rightarrow 1$ and $q_{n}^{n} \rightarrow c \in[0,1)$, as $n \rightarrow \infty$. Then, for any $f \in C_{x^{2}}^{*}[0, \infty)$ such that $f^{\prime}, f^{\prime \prime} \in C_{x^{2}}^{*}[0, \infty)$ and for a constant $A>0$ the following conclusion verify

$$
\lim _{n \rightarrow \infty}[n]_{q_{n}}\left(R_{n, q_{n}, a, b}(f ; x)-f(x)\right)=(c-1) x f^{\prime}(x)+\frac{1}{2}\left((1-c) x^{2}+x\right) f^{\prime \prime}(x),
$$

uniformly on $[0, A]$.

Proof. Assume that $x \in[0, \infty)$ and $f, f^{\prime}, f^{\prime \prime} \in C_{x^{2}}^{*}[0, \infty)$. By Taylor's formula, then one has

$$
f(t)=f(x)+(t-x) f^{\prime}(x)+\frac{1}{2}(t-x)^{2} f^{\prime \prime}(x)+\lambda(t ; x)(t-x)^{2} .
$$

Here, $\lambda(t ; x)$ is a Peano of the remainder term and since $\lambda(. ; x) \in C_{x^{2}}^{*}[0, \infty)$ then, $\lim _{t \rightarrow x} \lambda(t ; x)=0$. Operating $R_{n, q_{n}, a, b}(. ; x)$ to (15)

$$
\begin{aligned}
{[n]_{q_{n}}\left(R_{n, q_{n}, a, b}(f ; x)-f(x)\right) } & =[n]_{q_{n}} R_{n, q_{n}, a, b}((t-x) ; x) f^{\prime}(x)+\frac{1}{2}[n]_{q_{n}} R_{n, q_{n}, a, b}\left((t-x)^{2} ; x\right) f^{\prime \prime}(x) \\
& +[n]_{q_{n}} R_{n, q_{n}, a, b}\left(\lambda(t ; x)(t-x)^{2} ; x\right) .
\end{aligned}
$$

Utilizing the Cauchy-Schwarz inequality to the last term of above equality, then

$$
R_{n, q_{n}, a, b}\left(\lambda(t ; x)(t-x)^{2} ; x\right) \leq \sqrt{R_{n, q_{n}, a, b}\left(\lambda^{2}(t ; x) ; x\right)} \sqrt{R_{n, q_{n}, a, b}\left((t-x)^{4} ; x\right)} .
$$

It can be seen that as $\lambda(t ; x) \in C_{x^{2}}^{*}[0, \infty)$, thus from Theorem $1, \lim _{t \rightarrow x} \lambda(t ; x)=0$. Then, we find

$$
\lim _{n \rightarrow \infty} R_{n, q_{n}, a, b}\left(\lambda^{2}(t ; x) ; x\right)=\lambda^{2}(x ; x)=0 .
$$

Combining (16)-(17) and using Lemma 4, we get

$$
\lim _{n \rightarrow \infty}[n]_{q_{n}} R_{n, q_{n}, a, b}\left(\lambda(t ; x)(t-x)^{2} ; x\right)=0
$$

uniformly $[0, A]$. Consequently, we obtain the desired result as below:

$$
\lim _{n \rightarrow \infty}[n]_{q_{n}}\left(R_{n, q_{n}, a, b}(f ; x)-f(x)\right)=(c-1) x f^{\prime}(x)+\frac{1}{2}\left((1-c) x^{2}+x\right) f^{\prime \prime}(x) .
$$

\section{Some plots}

In this section, we give the comparison of the convergence of operators (3) to the function $f(x)=2 e^{-\frac{x}{5}} /(1+x)$ with the different values of $n, q, a, b$ parameters.

In Figure 1a, we compare the convergence of operators (3) to the function $f$ by keeping the parameters $n, a, b$ constant and increasing the values of $q$. In view of $0<q<1$, it can be seen that as the value of $q$ increases then the convergence of the operators (3) to $f$ becomes better.

In Figure $1 \mathrm{~b}$, we compare the convergence of operators (3) to the function $f$ by keeping the parameters $q, a, b$ constant 
and increasing the values of $n$. Since the values of $n$ increases, it is clear that the convergence of the operators (3) to the $f$ has become better.

Lastly, in Figure 1c, we compare the convergence of operators (3) to the function $f$ by keeping the parameters $n, q$ constant and increasing the values of $a$ and $b$. We have seen that; if we choose the natural numbers $a$ and $b$ very close to each other and large, in this case the convergence of the operators (3) to the $f$ becomes better.

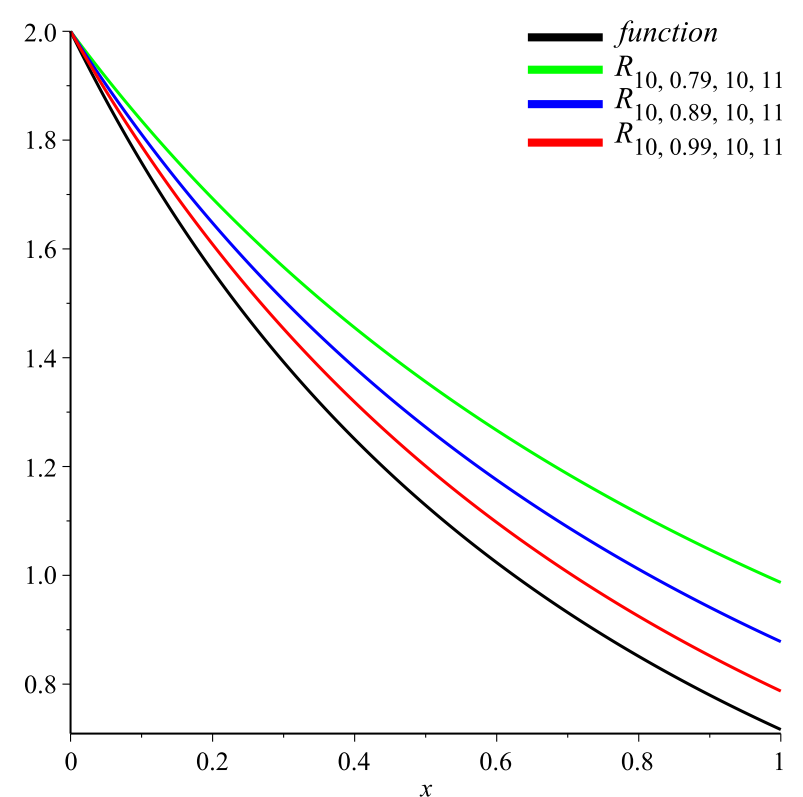

(a)

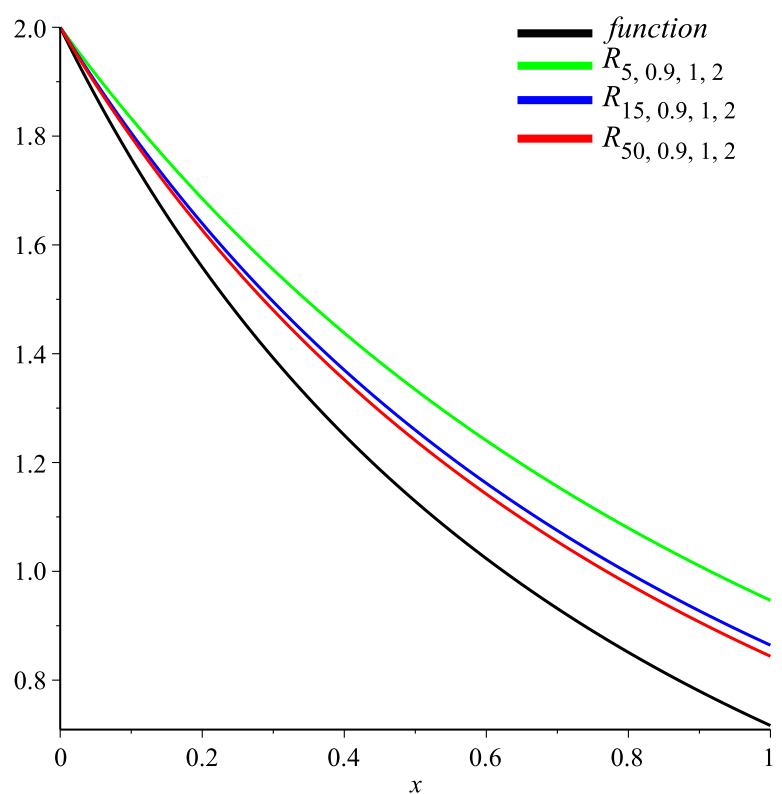

(b)

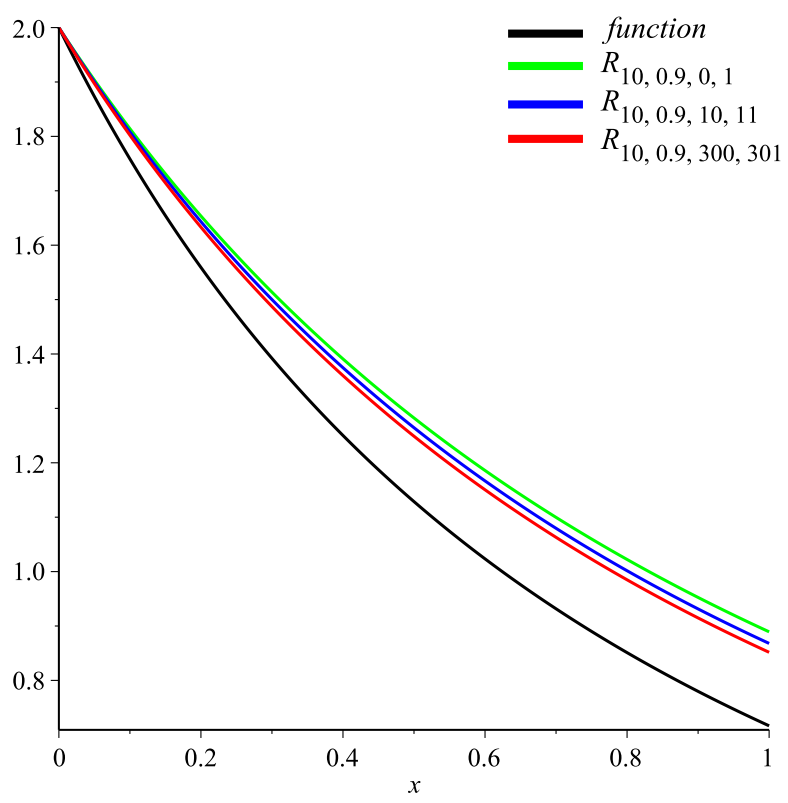

(c)

Fig. 1: The convergence of $R_{n, q, a, b}(f ; x)$ operators to $f(x)=2 e^{-\frac{x}{5}} /(1+x)$ under the different parameters. 


\section{Conclusion}

In this paper, we constructed a generalization of Szász-Mirakjan operators based on $q$ - integers. We obtained some preliminaries such as moments, central moments and uniform convergence of these operators. Moreover, we computed the order of approximation with the help of the modulus of continuity on a finite closed set and for the function belong to the Lipschitz class. Also, we investigated the local and weighted approximation properties on an unbounded interval and derived the Voronovskaya-type asymptotic theorem. Finally, with the help of Maple software, we compared the convergence of these operators to the certain function for the different values of $a, b, n$ and $q$.

\section{Acknowledgment}

The author is extremely thankful to the reviewers for critical reading of the manuscript leading to better presentation of the paper.

\section{Competing interests}

The authors declare that they have no competing interests.

\section{Authors' contributions}

All authors have contributed to all parts of the article. All authors read and approved the final manuscript.

\section{References}

[1] Acar, T. Quantitative $q$-Voronovskaya and $q$-Grüss-Voronovskaya-type results for $q$-Szász operators. Georgian Math. J., 23, 459-468, 2016.

[2] Acu, A.M. Bărbosu, D. and Sofonea, D.F. Note on a $q$-analogue of Stancu-Kantorovich operators. Miskolc Math. Notes, $16,2015$.

[3] Agrawal, P.N. Karsli, H. and Goyal, M. Szász-Baskakov type operators based on q-integers. J. Inequal. Appl., 1, 1-18, 2014.

[4] Ahasan, Mohd. and Mursaleen, M. Generalized Szász-Mirakjan type operators via $q$-calculus and approximation properties. Appl. Math. Comput., 371, 124916, 2020.

[5] Alotaibi, A. and Mursaleen M. Dunkl generalization of $q$-Szász-Mirakjan-Kantrovich type operators and approximation. J. Comput. Anal. Appl., 27, 66-76, 2019.

[6] Altomare, F. and Campiti, M. Korovkin-type approximation theory and its applications. vol. 17, Walter de Gruyter, 2011.

[7] Aral, A. A generalization of Szász-Mirakyan operators based on q-integers. Math. Comput. Modelling, 47, 1052-1062, 2008.

[8] Aral, A. and Gupta, V. The $q$-derivative and applications to $q$-Szász-Mirakyan operators. Calcolo, 43, 151-170, 2006.

[9] Aral, A. Ulusoy, G. and Deniz, E. A new construction of Szász-Mirakyan operators. Numer. Algorithms, 77, 313-326, 2017.

[10] Aslan, R. and Izgi, A. Agirlikli Uzaylarda q-Szasz-Kantorovich-Chlodowsky operatorlerinin yaklasimlari. Erciyes Universitesi Fen Bilimleri Enstitusu Fen Bilimleri Dergisi, 36, 137-149, 2020.

[11] Bascanbaz-Tunca, G. Erencin, A. and Tasdelen, F. On a sequnece of Kantorovich type operators via riemann type $q$-integral. Bull. Korean Math. Soc., 51, 303-315, 2014.

[12] Cai, Q.B. Zeng, X.M. and Cui, Z. Approximation properties of the modification of Kantorovich type $q$-Szász operators. J. Comput. Anal. Appl., 15, 176-187, 2013.

[13] Chen, S.N. Cheng, W.T. and Zeng, X.M. Stancu type generalization of modified gamma operators based on $q$-integers. Bull. Korean Math. Soc., 54, 359-373, 2017.

[14] Cheney, W.E. Introduction to approximation theory. Chelsea, New York, 1966.

[15] Devore, R.A. and Lorentz, G.G. Constructive Approximation. Springer, Heidelberg, 1993. 
[16] Gadzhiev, A.D. The convergence problem for a sequence of positive linear operators on unbounded sets, and theorems analogous to that of P.P. Korovkin. Dokl. Akad. Nauk, 218, 1001-1004, 1974.

[17] Gupta, V. and Pant, R.P. Rate of convergence for the modified Szász-Mirakyan operators on functions of bounded variation. J. Math. Anal. Appl., 233, 476-483, 1999.

[18] İspir, N. and Atakut, Ç. Approximation by modified Szász-Mirakyan operators on weighted spaces. Proc. Math. Sci., 112, 571-578, 2002.

[19] Kac, V. and Cheung, P. Quantum calculus. Springer Science \& Business Media, 2001.

[20] Lupas, A. A $q$-analogue of the Bernstein operator. In: Seminar on Numerical and Statistical Calculus. University of Cluj-Napoca, vol.9, 85-92, 1987.

[21] Mahmudov, N.I. On q-parametric Szász-Mirakyan operators. Mediterr. J. Math., 7, 297-311, 2010.

[22] Mirakjan, G.M. Approximation of continuous functions with the aid of polynomials. In Dokl. Acad. Nauk SSSR, 31, 201-205, 1941.

[23] Mursaleen, M. Al-Abied, A. and Ansari, K.J. On approximation properties of Baskakov-Schurer-Szász-Stancu operators based on $q$-integers. Filomat, 32, 1359-1378, 2018.

[24] Ousman, N. and Izgi, A. Szász operatorlerinin bir genellestirmesi. In: Abstracts of the 32nd National Mathematics Symposium, Ondokuz Mayis University, Samsun, Turkey, 31 August-3 September 2019.

[25] Phillips, G.M. Bernstein polynomials based on the $q$-integers. Ann. Numer. Math., 4, 511-518, 1997.

[26] Szász, O. Generalization of the Bernstein polynomials to the infinite interval. J. Res. Nat. Bur. Stand., 45, 239-245, 1950.

[27] Videnskii, V.S. On some classes of $q$-parametric positive linear operators. Selected topics in complex analysis, 213-222, BirkhÃ ouser, Basel, 2005. 